\title{
Experimental study of gas-water elongated bubble flow during production logging
}

\author{
Lu Jing ${ }^{1,2}$ and Wu Xiling, ${ }^{1,2 *}$ \\ ${ }^{1}$ Key Laboratory of Earth Prospecting and Information Technology, China University of Petroleum, Beijing 102249, China \\ ${ }^{2}$ State Key Laboratory of Petroleum Resource and Prospecting, China University of Petroleum, Beijing 102249, China \\ (C) China University of Petroleum (Beijing) and Springer-Verlag Berlin Heidelberg 2011
}

\begin{abstract}
In order to improve the interpretation of production log data on gas-water elongated bubble (EB) flow in horizontal wells, a multi-phase flow simulation device was set up to conduct a series of measurement experiments using air and tap water as test media, which were measured using a real production logging tool (PLT) string at different deviations and in different mixed flow states. By understanding the characteristics and mechanisms of gas-water EB flow in transparent experimental boreholes during production logging, combined with an analysis of the production log response characteristics and experimental production logging flow pattern maps, a method for flow pattern identification relying on $\log$ responses and a drift-flux model were proposed for gas-water EB flow. This model, built upon experimental data of EB flow, reveals physical mechanisms of gas-water EB flow during measurement processing. The coefficients it contains are the specific values under experimental conditions and with the PLT string used in our experiments. These coefficients also reveal the interference with original downhole flow patterns by the PLT string. Due to the representativeness that our simulated flow experiments and PLT string possess, the model coefficients can be applied as empirical values of logging interpretation model parameters directly to real production logging data interpretation, when the measurement circumstances and PLT strings are similar.
\end{abstract}

Key words: Horizontal wells, elongated bubble flow, flow patterns identification, drift-flux model, logging interpretation model

\section{Introduction}

Gas-water elongated bubble (EB) flow is one of the most common flow patterns that occur during production logging in horizontal wells. At the horizontal or uphill intervals of horizontal wells, gas bubbles (or plugs) that flow along the top of boreholes at a higher velocity relative to the continuous water phase are deformed due to gravitational differentiation and then form the so-called elongated bubble flow. So, the velocity and concentration profiles of gas-water mixed flow lose symmetry, and studies of its flow characteristics are obviously more complex than those of vertical flow $(\mathrm{Wu}$, 2004). Identifying flow patterns by effective methods and then choosing advanced practical logging interpretation models corresponding to different flow patterns can help interpret production $\log$ data qualitatively and quantitatively, thus helping gain an insight into fluid production intervals, real rates of gas-water two-phase fluids and development levels of reservoirs, so that the deliverability of horizontal wells can be enhanced to their maximum extent.

In the past two or three decades, a great many models

*Corresponding author. email: wuxiling@sina.com

Received May 4, 2010 based on experimental and theoretical studies have been developed to predict flow pattern transitions and flow parameters for two-phase flow in vertical or highly deviated circular pipes (Gomez et al, 2000; Hasan and Kabir, 2007; Hasan et al, 2007; Jepson and Taylor, 1993; Kaya et al, 2001). The hydrodynamic achievements in such studies provide a theoretical basis on which to develop production log data interpretation techniques for vertical or highly deviated wells. However, with the emergence of horizontal wells, the production log interpretation techniques applied in vertical or highly deviated wells are no longer adequate to deal with production log data interpretation issues arising from the complex states of flow in horizontal wells. Therefore, the reliability of results from production logging in horizontal wells is affected. In addition, most of the recent hydrodynamic achievements in research on horizontal or inclined pipes (de Sampaio et al, 2008; Ullmann and Brauner, 2006; Vuong et al, 2009; Wang et al, 2008; Zhang et al, 2009) require further attention to those flow parameters that cannot be obtained during production logging, and the solution usually needs complex iterative numerical calculations, so these achievements have not be applied to analyze and interpret production log data directly.

In this paper, the mixed fluids flow characteristics during 
production logging and processing are investigated, based on gas-water EB flow experimental measurements. At the same time, the production log response characteristics are analyzed to propose a $\log$ response identification method for understanding gas-water EB flow in horizontal wells. The drift-flux model for EB flow in horizontal wells was built up in light of our experimental data. On this basis, the way to determine the interpretation model parameters is found when this drift-flux model is used as the logging interpretation model to interpret real production logging data about EB flow in horizontal wells.

\section{Production logging experiments on simulated flow}

The multi-phase flow simulation device is represented in Fig.1. Experiments were made in No. II transparent experimental borehole (transparent, length $L=16 \mathrm{~m}$, inner diameter $\left.I D_{I I}=0.124 \mathrm{~m}\right)$. Interfacial tension between the liquid (tap water with a density $\rho_{\mathrm{w}}$ of $999 \mathrm{~kg} / \mathrm{m}^{3}$ at $16{ }^{\circ} \mathrm{C}$, under $101.3 \times 10^{3} \mathrm{~Pa}$ ) and the gas (air with a density $\rho_{\mathrm{g}}$ of $1.22 \mathrm{~kg} / \mathrm{m}^{3}$ at $16{ }^{\circ} \mathrm{C}$, under $\left.101.3 \times 10^{3} \mathrm{~Pa}\right) \sigma_{\text {wg }}$ is $73.3 \times 10^{-3} \mathrm{~N} /$ $\mathrm{m}$. Steady-state mixed gas-water flow was measured by a PLT string, which consists of a caged fullbore flowmeter (CFB), radioactive fluid density tool (FDR), capacitance water holdup tool (CWH), capacitance array tool (CAT) and a quartz pressure \& casing collar locator tool (QTC including the temperature \& pressure of quartz ), made by Sondex. The general purpose of this experimental effort is to collect a maximum amount of data on gas-water EB flow in horizontal wells under the maximum fluids rates that the experimental system can provide, so the experimental matrix covers a wide range of flow rates for each phase, at deviations $\theta$ (angle from vertical) $75^{\circ}, 85^{\circ}$ and $90^{\circ}$ respectively. The total air-water flow rates $Q_{\mathrm{T}}$ were $50,100,200,400$ and $800 \mathrm{~m}^{3} / \mathrm{d}$, and the water cuts $C_{\mathrm{W}}$ were $0,10 \%, 30 \%, 50 \%, 70 \%, 90 \%$ (except for $Q_{\mathrm{T}}=800 \mathrm{~m}^{3} / \mathrm{d}$ ), respectively. There were twenty-nine experiments at each deviation, and in each of the experiment four kinds of measurements had to be done successively when the mixed flow reached a steady state: a) observe and record flow patterns; b) measure the actual inlet flow rates $Q_{\mathrm{g}}^{\prime}, Q_{\mathrm{w}}^{\prime}$; temperature $T$ and pressure $P$ of two phase fluids; c) dynamically measure the mixed fluid with the PLT string in boreholes; d) conduct the steady-state shut-in for calculating the shut-in water holdup after the above log measurements. Towards the end, eighty-seven logging experiments in different flow conditions had been conducted. In each of the measuring experiments, the above-mentioned four data are collected.

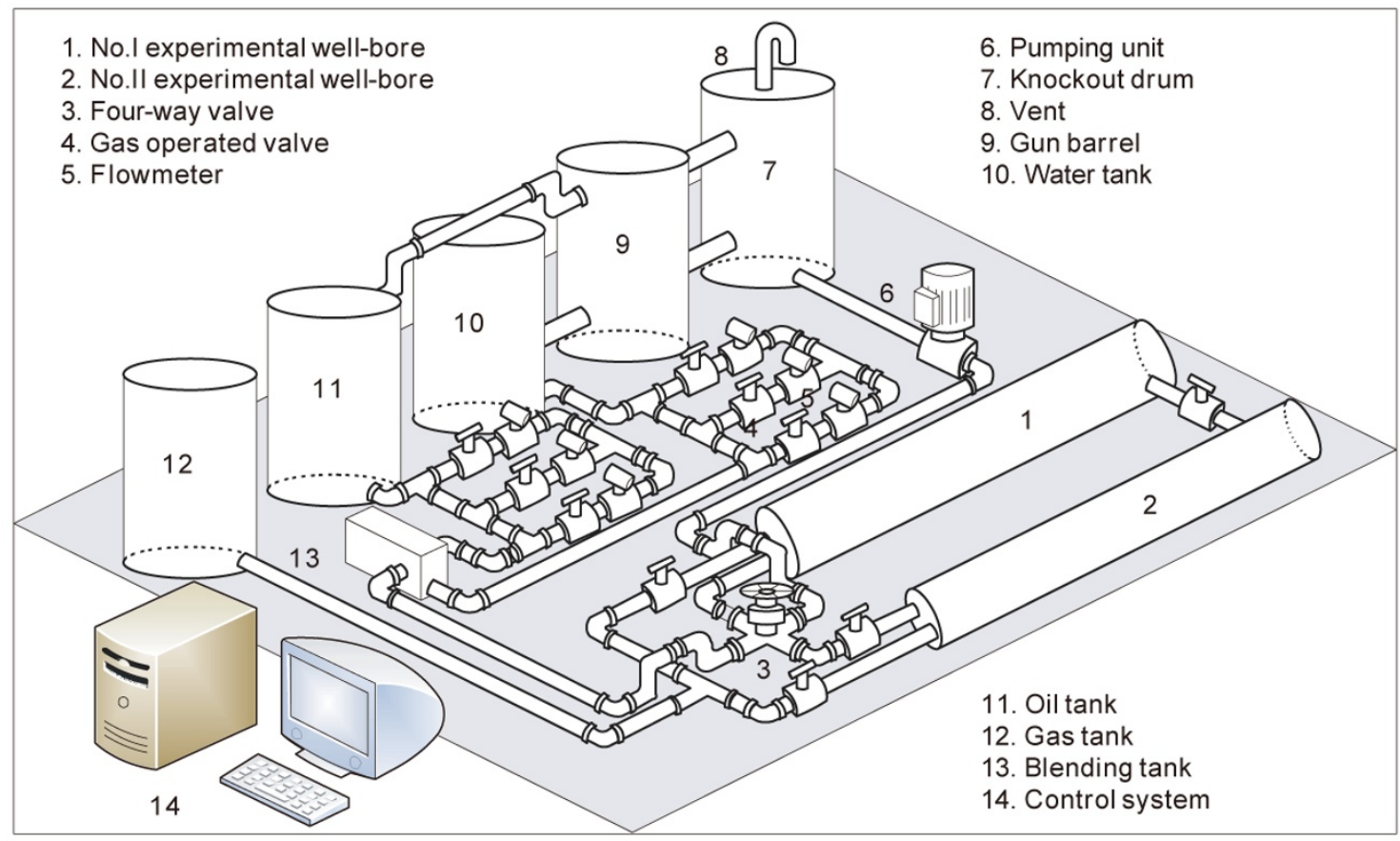

Fig. 1 Schematic of the multi-phase flow simulation device

\section{Gas-water EB flow identification by $\log$ response}

\subsection{Analysis of the production logging flow patterns}

During our production logging simulation experiments, there are four kinds of flow patterns in Fig. 2, as observed in the No. II transparent borehole. They are stratified smooth flow (SS), stratified wave flow (SW), elongated bubble flow (EB) and slug flow (SL) respectively. Actual inlet rates of gas and water $Q_{\mathrm{g}}^{\prime}, Q_{\mathrm{w}}^{\prime}$ measured in experiments were used to calculate the superficial velocity $v_{\mathrm{sg}}, v_{\mathrm{sw}}$ of gas and water according to the definitions: $v_{\mathrm{sg}}=Q_{\mathrm{g}}^{\prime} / A, v_{\mathrm{sw}}=Q^{\prime}{ }_{\mathrm{w}} / A$ ( $\left.A=\pi \cdot I D_{\mathrm{II}}^{2} / 4\right)$. Together with flow patterns observed in the above-said borehole, the production logging flow pattern maps at different deviations were illustrated in Fig. 3. 


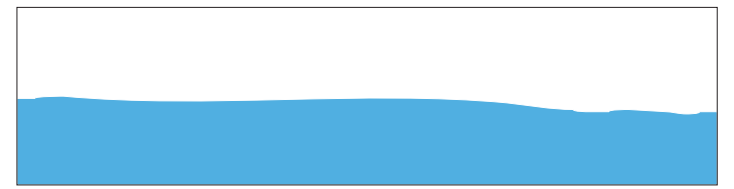

Stratified smooth flow(SS)

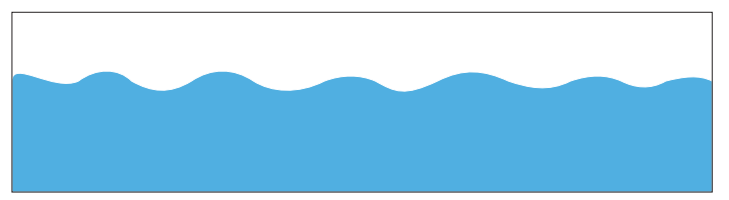

Stratified wave flow(SW)

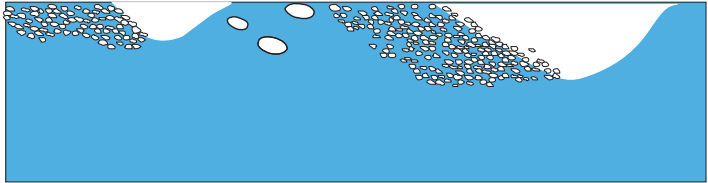

Elongated bubble flow(EB)

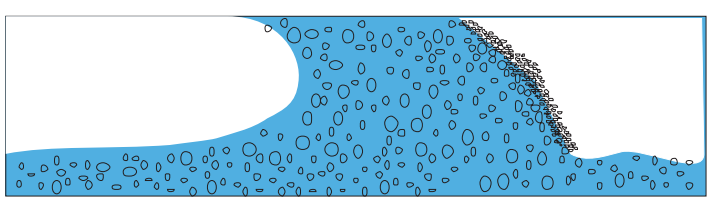

Slug flow(SL)

Fig. 2 Experimental flow patterns in production logging experiments on simulation flow
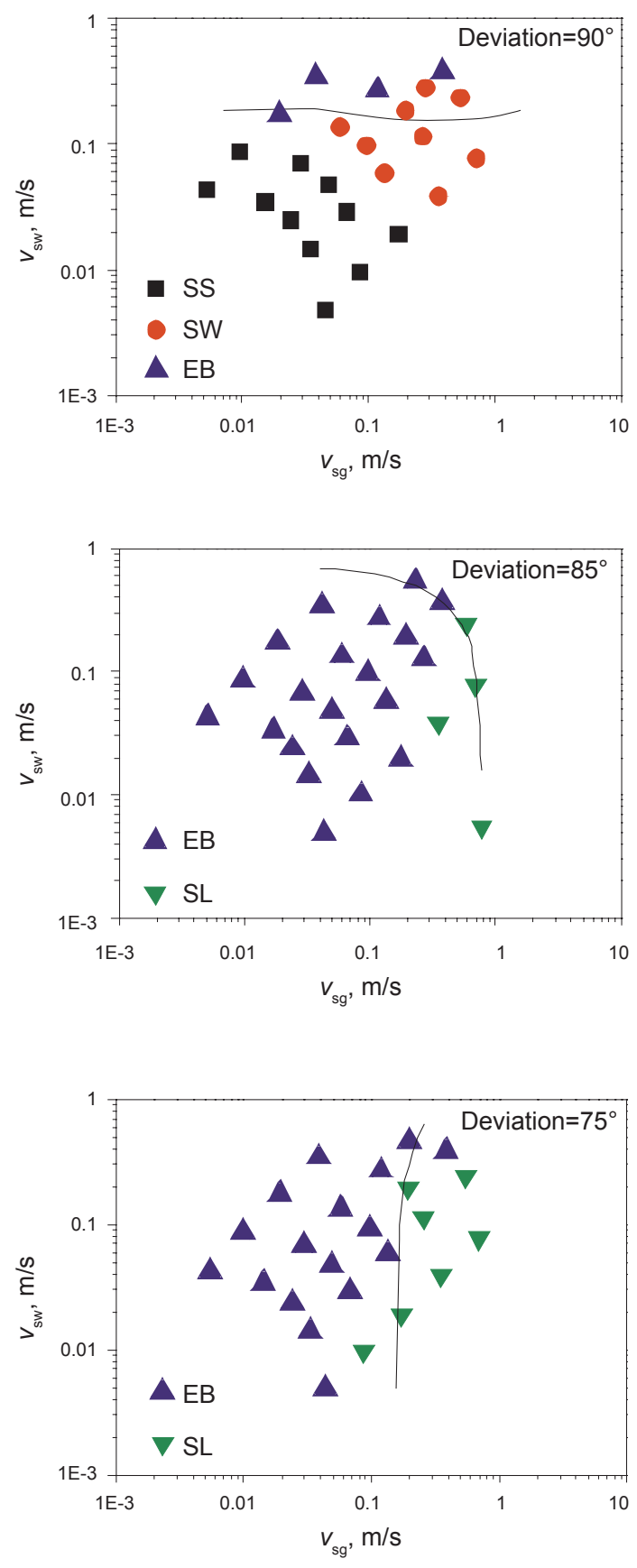

Fig. 3 Production logging flow pattern maps with hydrodynamic transition boundaries
In order to analyze the characteristics of gas-water twophase mixed flow during PLT string measurement and processing, results concluded from hydrodynamic studies without intrusive measurement tools were applied to compare with the production logging flow pattern maps of the experiments. In these maps, EB flow pattern transition boundaries corresponding to the experiment deviations were cited (Barnea et al, 1985; Kokal and Stanislav, 1989). The distribution trends of the experimental data points on production logging flow patterns are similar to the above hydrodynamic achievements. This indicates that the gas-water mixed flow in production logging still obeys the general laws of hydrodynamics, but the changes also show the interferences with the mixed flow patterns caused by the PLT string measurement, which gives gas-water flow at measured intervals of some unique distribution characteristics ( $\mathrm{Lu}$ et al, 2010). Specifically, in our production logging experiments, $\mathrm{SS} / \mathrm{SW}$ flow would transform into EB flow, when the $Q_{\mathrm{w}}$ exceeds $156 \mathrm{~m}^{3} / \mathrm{d}$ to $208 \mathrm{~m}^{3} / \mathrm{d}$ in boreholes at an deviation (horizontal) angle of $90^{\circ}$, whose inner diameter ID is $0.124 \mathrm{~m}$; EB flow would not transform into SL flow until $Q_{\mathrm{T}}$ is higher than $400 \mathrm{~m}^{3} / \mathrm{d}$ and $C_{\mathrm{w}}$ is lower than $30 \%$ in the above similar boreholes deviated at $85^{\circ}$; while when the deviation angle was $75^{\circ}$ (borehole ID $=0.124 \mathrm{~m}$ ), EB flow transform into SL just when $Q_{\mathrm{T}}$ was $100 \mathrm{~m}^{3} / \mathrm{d}$ to $200 \mathrm{~m}^{3} / \mathrm{d}, C_{\mathrm{w}}$ lower than $10 \%$ and $Q_{\mathrm{T}}$ higher than $200 \mathrm{~m}^{3} / \mathrm{d}$ but $C_{\mathrm{w}}$ is lower than $30 \%$. The flow pattern distribution characteristics known from simulation flow logging experiments can assist our downhole flow pattern identification to ensure that the applicable logging interpretation models can be chosen for different individual flow patterns, so that the quantitative logging interpretation accuracy can be improved.

\subsection{Analysis of the production log response characteristic}

By observing and identifying the flow patterns in transparent experimental boreholes during our experimental measurements, and by analyzing the characteristics of corresponding production log responses, it is clearly seen that gas-water EB flow can be identified quickly in light of the fluctuation of the CFB log response, when sudden changes in such factors, as unstable cable speed and the downhole string inner diameter have been excluded. 
Because of the gravitational differentiation that occurs in fluids of different densities in horizontal wells, bubbles (plugs) flowing in a continuous water phase deform, like Fig. 4. Those bubbles (plugs) with a higher velocity disturb the normal water on the gas-water interface when they float upward. This phenomenon leads to a water phase reflux near the gas-water interface. Thus the spinner, whose chief act is to mix fluids across the borehole, is driven by three rotary moments simultaneously. First of all, there are two kinetic moments respectively resulting from gas and water two-phase fluids that flow upward along the borehole. Secondly, there is still a negative moment resulting from the reflux near the gaswater interface. Also, because the gas density and viscosity are considerably smaller than in the water phase, its kinetic moment is correspondingly lower than that of the water phase, plus the negative moment resulting from the water reflux near the gas-water interface, and the spinner's speed would slow down when the central CFB encounters elongated bubbles. Thus, EB flow can be identified intuitively by the fluctuation of CFB logs. Additionally, it is worth noting that the fluctuation is not so intense that the spinner rotates in an opposite way as it acts in SL flow.

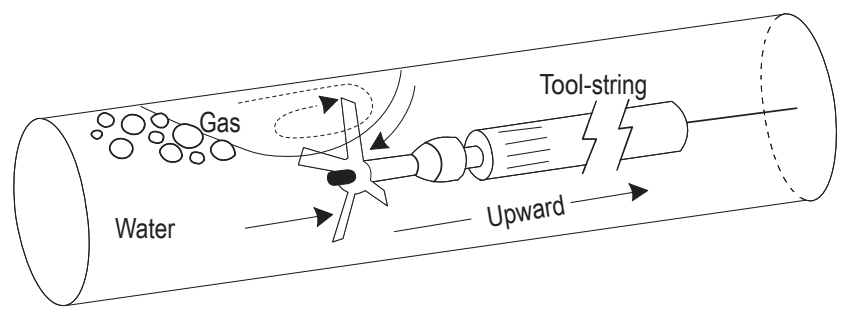

Fig. 4 Principle of the caged fullbore flowmeter $\log$ response characteristics of elongated bubble flow

\section{Logging data interpretation model}

Knowing gas-water mixed flow states in horizontal wells, and proposing different interpretation models that are applied to different individual flow patterns, are the foundation upon which to improve the accuracy of interpretation of production logging information. Based on the analysis of gas-water EB flow experimental logging flow patterns and flow patterns identification by log responses, an advanced and practical logging interpretation model will be proposed in this section, which reveals the physical mechanism of EB flow in horizontal wells.

\subsection{Hydrodynamic model}

Under horizontal or near-horizontal conditions, it can be observed in experiments that elongated bubbles are distributed in a continuous water phase. Under the action of buoyancy, elongated bubbles of a higher velocity tend to float along top of the pipeline and form the EB flow. The drift-flux model, like that described in Eq.(1), is adequate to describe the physical mechanisms of slip between air and water in EB flow.

$$
\frac{v_{\mathrm{sg}}}{H_{\mathrm{g}}}=C_{0} v_{\mathrm{m}}+v_{\mathrm{d}}
$$

where $C_{0}$ is the concentration coefficient, 1, showing the degree of non-uniformity of velocity and concentration profiles; $v_{\mathrm{m}}$ is the mean velocity of mixed fluids, $\mathrm{m} / \mathrm{s} ; v_{\mathrm{d}}$ is the drift velocity, $\mathrm{m} / \mathrm{s}$, showing the velocity difference caused by the effect of buoyancy between light and heavy phases; $H_{\mathrm{g}}$ is the gas holdup, showing the value of gas cuts in the mixed flow cross-section. We established the physical model like Fig.5 to develop the drift velocity $v_{\mathrm{d}}$ expression applicable to gas-water EB flow in horizontal wells.

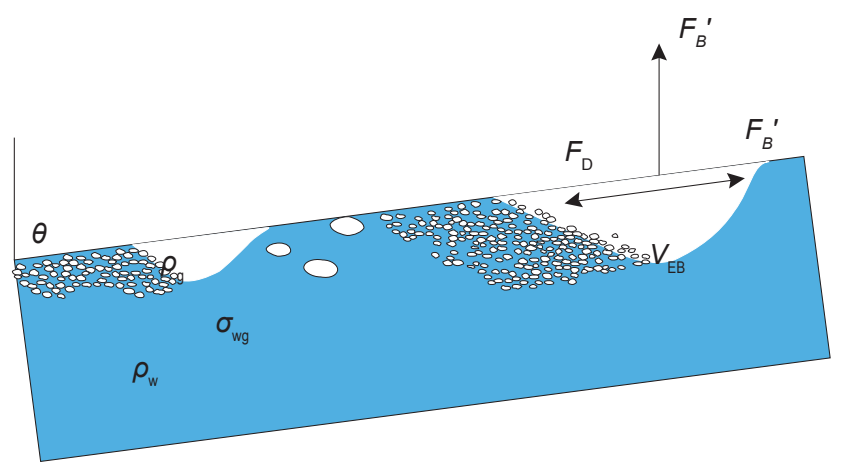

Fig. 5 Physical model for gas-water EB flow in horizontal wells

The terminal rise velocity of elongated bubble $v_{\infty}, \mathrm{m} /$ $\mathrm{s}$, which shows the rise velocity of the elongated bubbles in an inclined static fluid column, is just the drift velocity $v_{\mathrm{d}}$ in Eq.(1), m/s. It can be evaluated by considering the drag and buoyancy forces acting on a single elongated bubble under the assumption that bubbles do not interfere with each other.

For a single bubble, the projection $F_{\mathrm{B}}^{\prime}$ of the buoyancy $F_{\mathrm{B}}$ along the inclined pipeline can be expressed as (Kaya et al, 2001)

$$
F_{\mathrm{B}}^{\prime}=F_{\mathrm{B}} \cos \theta=\left(\rho_{\mathrm{m}}-\rho_{\mathrm{g}}\right) V_{\mathrm{EB}} \mathrm{g} \cos \theta
$$

The drag force $F_{\mathrm{D}}$ can be expressed as

$$
F_{\mathrm{D}}=A_{\mathrm{P} \theta} C_{\mathrm{D} \theta} \rho_{\mathrm{w}} v_{\infty \theta}^{2} / 2
$$

In Eq.(2) $F_{\mathrm{B}}$ is the buoyancy of a single elongated bubble, $\mathrm{N} ; F_{\mathrm{B}}{ }^{\prime}$ is the projection force of $F_{\mathrm{B}}$ along the inclined pipeline, $\mathrm{N} ; \theta$ is the well deviation (angle from vertical) of the pipeline, degree; $\rho_{\mathrm{m}}$ is the mixed fluid density, $\mathrm{kg} / \mathrm{m}^{3}$, given by the gas density $\rho_{\mathrm{g}}, \mathrm{kg} / \mathrm{m}^{3}$, the

$$
\rho_{\mathrm{m}}=\rho_{\mathrm{g}} \cdot H_{\mathrm{g}}+\rho_{\mathrm{w}} H_{\mathrm{w}}=\rho_{\mathrm{g}} \cdot H_{\mathrm{g}}+\rho_{\mathrm{w}}\left(1-H_{\mathrm{g}}\right)
$$

$V_{\mathrm{EB}}$ is the volume of an elongated bubble. Under the assumption of a single bubble deforming but its volume remaining constant, the volume of elongated bubbles, whose inner diameter is $d, \mathrm{~m}$, can be given by a spherical volume

$$
V_{\mathrm{EB}}=\iint\left(z_{2}-z_{1}\right) \mathrm{d} x \mathrm{~d} y=\frac{4}{3} \pi\left(\frac{d}{2}\right)^{3}=\frac{\pi d^{3}}{6}
$$

In Eq.(3), $F_{\mathrm{D}}$ is the drag force, which occurs as the elongated bubble rises up, $\mathrm{N} ; A_{\mathrm{P} \theta}$ is the elongated bubble projection area, which is normal to its velocity, $\mathrm{m}^{2} ; C_{\mathrm{D} \theta}$ is the 
drag coefficient, 1 . Their multiple $A_{\mathrm{P} \theta} C_{\mathrm{D} \theta}$ has an experimental relationship with $A_{\mathrm{P} 0} C_{\mathrm{D} 0}$ in vertical pipelines as (Hasan and Kabir, 1988)

$$
\frac{A_{\mathrm{P} 0} C_{\mathrm{D} 0}}{A_{\mathrm{P} \theta} C_{\mathrm{D} \theta}}=(1+\sin \theta)^{2.4}
$$

The projection area $A_{\mathrm{P} 0}$ and drag coefficient $C_{\mathrm{D} 0}$ of bubbles in vertical pipes are given in the equations

$$
\begin{aligned}
& A_{P 0}=\pi d^{2} / 4 \\
& C_{\mathrm{D} 0}=0.575 \sqrt{\mathrm{g}\left(\rho_{\mathrm{w}}-\rho_{\mathrm{g}}\right) d^{2} / \sigma_{\mathrm{wg}}}
\end{aligned}
$$

where $\sigma_{\mathrm{wg}}$ is the interfacial tension between gas and water, N/ $\mathrm{m}$; thus the balance between the buoyancy and the drag force gives

$v_{\infty \theta}=C_{\mathrm{f}}\left[\frac{\mathrm{g} \sigma_{\mathrm{wg}}\left(\rho_{\mathrm{w}}-\rho_{\mathrm{g}}\right)}{\rho_{\mathrm{w}}^{2}}\right]^{0.25} \sqrt{\left(1-H_{\mathrm{g}}\right) \cdot \cos \theta}(1+\sin \theta)^{1.2}$

According to Eq.(1) and Eq.(9), the drift-flux model for gas-water EB flow in horizontal wells can be expressed as

$$
\begin{aligned}
& \frac{v_{\mathrm{sg}}}{H_{\mathrm{g}}}=C_{0} v_{\mathrm{m}} \\
& +C_{\mathrm{f}}\left[\frac{\mathrm{g} \sigma_{\mathrm{wg}}\left(\rho_{\mathrm{w}}-\rho_{\mathrm{g}}\right)}{\rho_{\mathrm{w}}^{2}}\right]^{0.25} \sqrt{\left(1-H_{\mathrm{g}}\right) \cdot \cos \theta}(1+\sin \theta)^{1.2} \\
& \frac{v_{\mathrm{sg}}}{H_{\mathrm{g}} \sqrt{1-H_{\mathrm{g}}}}=C_{0} \frac{v_{\mathrm{m}}}{\sqrt{1-H_{\mathrm{g}}}} \\
& +C_{\mathrm{f}}\left[\frac{\mathrm{g} \sigma_{\mathrm{wg}}\left(\rho_{\mathrm{w}}-\rho_{\mathrm{g}}\right)}{\rho_{\mathrm{w}}^{2}}\right]^{0.25} \sqrt{\cos \theta}(1+\sin \theta)^{1.2}
\end{aligned}
$$

where we call the $C_{\mathrm{f}}$. a drift velocity coefficient, showing the degree of velocity difference between the gas phase and the water phase.

\subsection{Experimental data assessment}

Experimental data should be processed before they are used to assess the above hydrodynamic model (Eq.(10) or Eq.(10')). With the definition of mean velocity $v_{\mathrm{m}}=Q_{\mathrm{T}}{ }^{\prime} / A$ (where $A=\pi \cdot I D_{\mathrm{II}}^{2} / 4$ ), the actual total gas-water rate $Q_{\mathrm{T}}{ }^{\prime}\left(Q_{\mathrm{T}}{ }^{\prime}=Q_{g}+Q_{\mathrm{w}}{ }^{\prime}\right)$ is used to calculate the mean velocity of the mixed fluid $v_{\mathrm{m}}$ in No. II experimental borehole. Furthermore, the production log responses of FDR, CWH and CAT are used to calculate the density water holdup, the capacitance water holdup and the CAT water holdup.
A comprehensive water holdup $H_{\mathrm{w}}$, which results from the above calculated water holdups and shut-in water holdup $\left(H_{\mathrm{g}}=1-H_{\mathrm{w}}\right)$, is analyzed.

According to Eq.(10'), we established a coordinate system using $\frac{v_{\mathrm{m}}}{\sqrt{1-H_{\mathrm{g}}}}$ and $\frac{v_{\mathrm{sg}}}{H_{\mathrm{g}} \sqrt{1-H_{\mathrm{g}}}}$ as a horizontal axis and a vertical axis respectively, and linear regressed data points of each deviation illustrated in Fig.6. The results of our data assessment reveal that there are good linear relationships among the experimental data about different deviations. The change of deviations does not interfere with the regression lines parallel to each other. It is therefore clear that the gaswater EB flow experimental data obey the law of the model like Eq.(10) or Eq.(10'). Further calculations can help determine the model coefficients $C_{0}$ and $C_{\mathrm{f}}$.

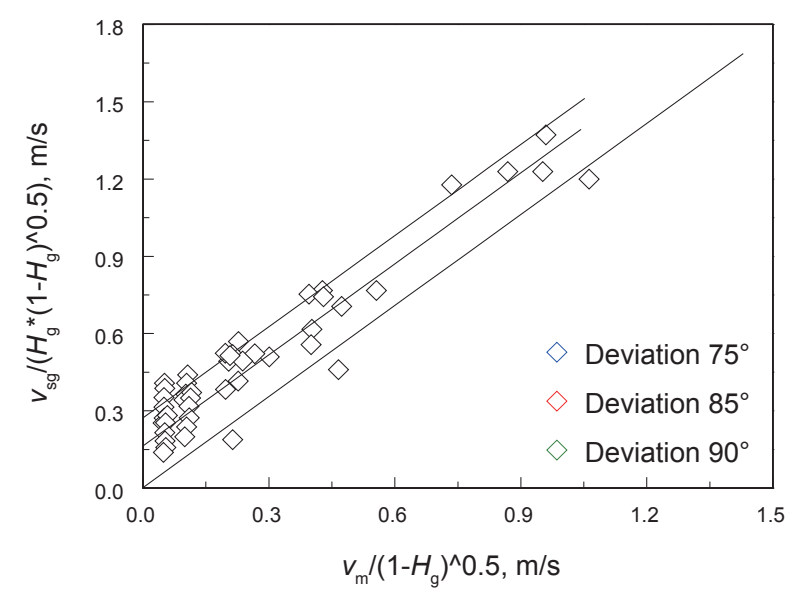

Fig. 6 Model assessment plate for the gas-water elongated bubble flow in horizontal wells

On the model assessment plate (Fig. 6), the slope of every line, which regresses linearly from experimental data under different well deviations, is 1.18 . So, it can be known from Eq. $\left(10^{\prime}\right)$ that $C_{0}$ is 1.18 . The vertical axis intercepts of each regression line at experimental well deviations of $75^{\circ}, 85^{\circ}$ and $90^{\circ}$ are $0.275 \mathrm{~m} / \mathrm{s}, 0.163 \mathrm{~m} / \mathrm{s}$ and 0 . It also can be known from Eq. (10') that these intercepts are values of the second term on the right side of the equation at the above experimental deviations. In this part, when the experimental fluid media and well deviations are

fixed, $\left[\frac{\mathrm{g} \sigma_{\mathrm{wg}}\left(\rho_{\mathrm{w}}-\rho_{\mathrm{g}}\right)}{\rho_{\mathrm{w}}^{2}}\right]^{0.25} \sqrt{\cos \theta}(1+\sin \theta)^{1.2} \quad$ must be a constant. In terms of our experiments, their numeric values are $0.188 \mathrm{~m} / \mathrm{s}, 0.111 \mathrm{~m} / \mathrm{s}$ and $0 \mathrm{~m} / \mathrm{s}$ respectively. Now, it can be calculated that the drift velocity coefficient $C_{\mathrm{f}}$ is 1.47 . Thus, the gas-water EB flow drift-flux model applicable to production logging interpretations in horizontal wells can be built up and expressed as

$$
\frac{v_{\mathrm{sg}}}{H_{\mathrm{g}}}=1.18 v_{\mathrm{m}}+1.47\left[\frac{\mathrm{g} \sigma_{\mathrm{wg}}\left(\rho_{\mathrm{w}}-\rho_{\mathrm{g}}\right)}{\rho_{\mathrm{w}}^{2}}\right]^{0.25} \sqrt{\left(1-H_{\mathrm{g}}\right) \cos \theta}(1+\sin \theta)^{1.2}
$$




\subsection{Logging interpretation model}

Because of gravitational differentiation the gas phase, which flows upward along the horizontal or near horizontal pipelines, is of a higher phase velocity than the water phase flowing at the bottom, so the mixed fluid velocity and concentration profiles are therefore sharper at the top of the pipelines. The motional measurement of PLT string in boreholes, especially the stirring effect of CFB spinner, results in mixed flow profiles that become flat and diminish the velocity difference between the two phases. Therefore, in the drift-flux model (Eq.(11)) proposed in our experimental study, the concentration coefficient $\mathrm{C}_{0}$, which is used to show the degree of the non-uniformity of the known flow profile, and the drift velocity coefficient $\mathrm{C}_{\mathrm{f}}$, which is used to show the degree of velocity difference between gas and water, are smaller than those empirical values (such as 1.2 and 1.53), which are derived from experimental studies without intrusive measurement tools in pipes.

The drift-flux model built is based on our experimental study, and its coefficients reveal the characteristics and physical mechanisms of EB flow in horizontal or near horizontal upward intervals. So, they are applicable to logging information interpretations in horizontal wells. The interactions between the production logging tools and the measured fluid, as well as the degree of change of the original downhole flow states, caused by the PLT string, all vary with each tool. Therefore, the selection and compound mode of the PLT string directly affects the mixed fluid flow. The above gas-water EB flow drift-flux model built upon our experimental study is based on the multi-phase flow simulation device and the PLT string selected in experiments, so if this model is used as a logging interpretation mode to interpret the real production logging data of $\mathrm{EB}$ flow in horizontal wells, the interpretation parameters $\mathrm{C}_{0}$ and $\mathrm{C}_{\mathrm{f}}$ need to be determined according to individual tests against different measurement circumstances and PLT strings. In view of the experimental borehole, the PLT string and its compound mode designed and used in our experiments have a certain representativeness in real production logging for horizontal wells. Therefore, if the measurement circumstances and PLT strings are similar, the coefficients determined from our experimental study can be used as empirical values of the production logging interpretation model parameters to interpret the real production logging data for EB flow in horizontal wells.

\section{Conclusion}

1) The similarities between our experimental production logging flow pattern maps and hydrodynamic achievements reveal that the gas-water mixed flow in PLT string measurements obey the general laws of hydrodynamics. But the movements of flow pattern boundaries show the influence of the PLT string measurement over the original downhole flow. These flow pattern maps can assist the identification of downhole flow states. In addition, EB flow in horizontal wells can be identified in view of the log response characteristics of the CFB quickly and intuitively, when such factors as unstable cable speed and sudden changes of the inner diameter of the downhole string have been excluded.

2) The gas-water EB flow drift-flux model built upon our experimental study reveals meanwhile the mixed flow physical mechanisms and the interference with the original downhole flow caused by the PLT string in horizontal wells. Combined with the parameters of the interpretation model, which are determined by individual tests, this driftflux model can be used as a logging interpretation model to interpret real production logging information. However, our model coefficients $\mathrm{C}_{0}$ and $\mathrm{C}_{\mathrm{f}}$, determined from experimental data, still can be used as an empirical value of the logging interpretation model parameters.

\section{References}

Barnea D, Shoham O, Taitel Y, et al. Gas-liquid Flow in Inclined Tubes: Flow pattern transitions for upward flow. Chemical Engineering Science. 1985. 40(1): 131-136

Gomez L E, Shoham O, Schmidt Z, et al. Unified Mechanistic Model for Steady-state Two-Phase Flow: Horizontal to Vertical Upward Flow. SPE Journal. 2000. 5(3): 339-350

Hasan A R and Kabir C S. Predicting Multiphase Flow Behavior in a Deviated Well. SPE Production Engineering. 1988. 3(4): 474-482

Hasan A R and Kabir C S. A Simple Model for Annular Two-phase Flow in Wellbores. SPE Production \& Operations. 2007. 22(2): 168-175

Hasan A R, Kabir C S and Sayarpour M. A Basic Approach to Wellbore Two-phase Flow Modeling. SPE Annual Technical Conference and Exhibition held in Anaheim, California, 11-14 November, 2007 (SPE paper 109868)

Jepson W P and Taylor R E. Slug Flow and Its Transitions in Largediameter Horizontal Pipes. International Journal of Multiphase Flow. 1993. 19(3): 411-420

Kaya A S, Sarica C and Brill J P. Mechanistic Modeling of Two-phase Flow in Deviated Wells. SPE Production \& Operations. 2001. 16(3): 156-165

Kokal S L and Stanislav J F. An Experimental Study of Two-phase Flow in Slightly Inclined Pipes--I. Flow patterns. Chemical Engineering Science. 1989. 44(3): 665-679

Lu J, Wu X L, Huang Z J, et al. Experimental Study of Horizontal Gaswater Flow Patterns during Production Well Logging. Science \& Technology Review. 2010. 28(3): 59-62 (in Chinese)

De Sampaio P A B, Faccini J L H and Su J. Modelling of Stratified Gasliquid Two-phase Flow in Horizontal Circular pipes. International Journal of Heat and Mass Transfer. 2008. 51(11-12): 2752-2761

Ullmann A and Brauner N. Closure Relationships of Two-fluid Models with Two-phase Smooth and Stratified Wavy Flows. International Journal of Multiphase Flow. 2006. 32(1): 82-105

Vuong D H, Zhang H-Q, Sarica C, et al. Experimental Study of High Viscosity Oil/Water Flow in Horizontal and Vertical Pipes. SPE Annual Technical Conference and Exhibition held in New Orleans, Louisiana, 4-7 October, 2009 (SPE paper 124542)

Wang C C, Chen I Y, Lin Y T, et al. A Visual Observation of the AirWater Two-phase Flow in Small-Diameter Tubes Subject to the Influence of Vertical Return Bends. Chemical Engineering Research and Design. 2008. 86(11): 1223-1235

Wu X L. Petroleum Production Logging Principles. Beijing: Higher Education Press. 2004. 66-126 (in Chinese)

Zhang L, Du W, Bi H T, et al. Gas-liquid Two-phase Flow Distributions in Parallel Channels for Fuel Cells. Journal of Power Sources. 2009. 189(2): 1023-1031

(Edited by Yang Lei) 